\title{
The unintended consequences of overinvestigating incidentalomas
}

\author{
Cite as: CMAJ 2017 August 21;189:E1070-1. doi: 10.1503/cmaj.161205
}

$\mathrm{n}$ the second month of my undergraduate degree, a bike crash left me with hip pain and a hindered ability to run. Anxious to return to triathlon training, I sought physiotherapy. The sports medicine clinic first sent me for an x-ray. And then a bone scan. And then more $x$-rays, blood tests, a magnetic resonance imaging (MRI) scan and computed tomography (CT) scans. I willingly obliged, because I believed that these tests would guide physiotherapy and diminish the pain in my hips.

I was wrong.

I can vividly remember the day before my physics final exam, six months after crashing my bike, when I was told that there was a hairline fracture in my pelvic bone pointing like an arrow to a tumour, with another tumour nearby - one was benign, the other active. The physics exam quickly became the least of my worries.

To 19-year-old me, "active tumour" sounded a lot like cancer. Google agreed. Sure enough, by the time I had finished my physics exam, there was a message on my phone with details for an appointment with an oncologist the very next day. I continued as an oncology patient for two and a half years before learning that the tumours were not cancer. I would have made different decisions throughout the first half of my undergraduate degree had I known that, despite all the fancy tests and appointments, I might be perfectly healthy. Not necessarily better decisions, but different ones, because my priorities changed once I started believing my future on this earth was limited.

Five years later in graduate school, I learned the official term for my accidentally found tumours - incidentalomas which are closely tied to the concept of overdiagnosis: the diagnosis of "disease" that will never cause symptoms or death in a patient's lifetime. However, at the time I did not know that you can have tumours that will never lead to symptoms, sickness or death. Looking back, I now understand that I experienced many of the unintended consequences of having benign incidentalomas overinvestigated.

After the first appointment with my oncologist, I avoided getting close to anyone, put very little effort toward school and
The consequences did not stop after my oncologist cleared me. For a while, I experienced what is probably best described as survivor's guilt. Why did my tumours stop growing when so many other people are not so fortunate? Soon I realized these feelings were not helpful to anyone. I concentrated instead on trying not to take anything for granted and working hard at any opportunities I

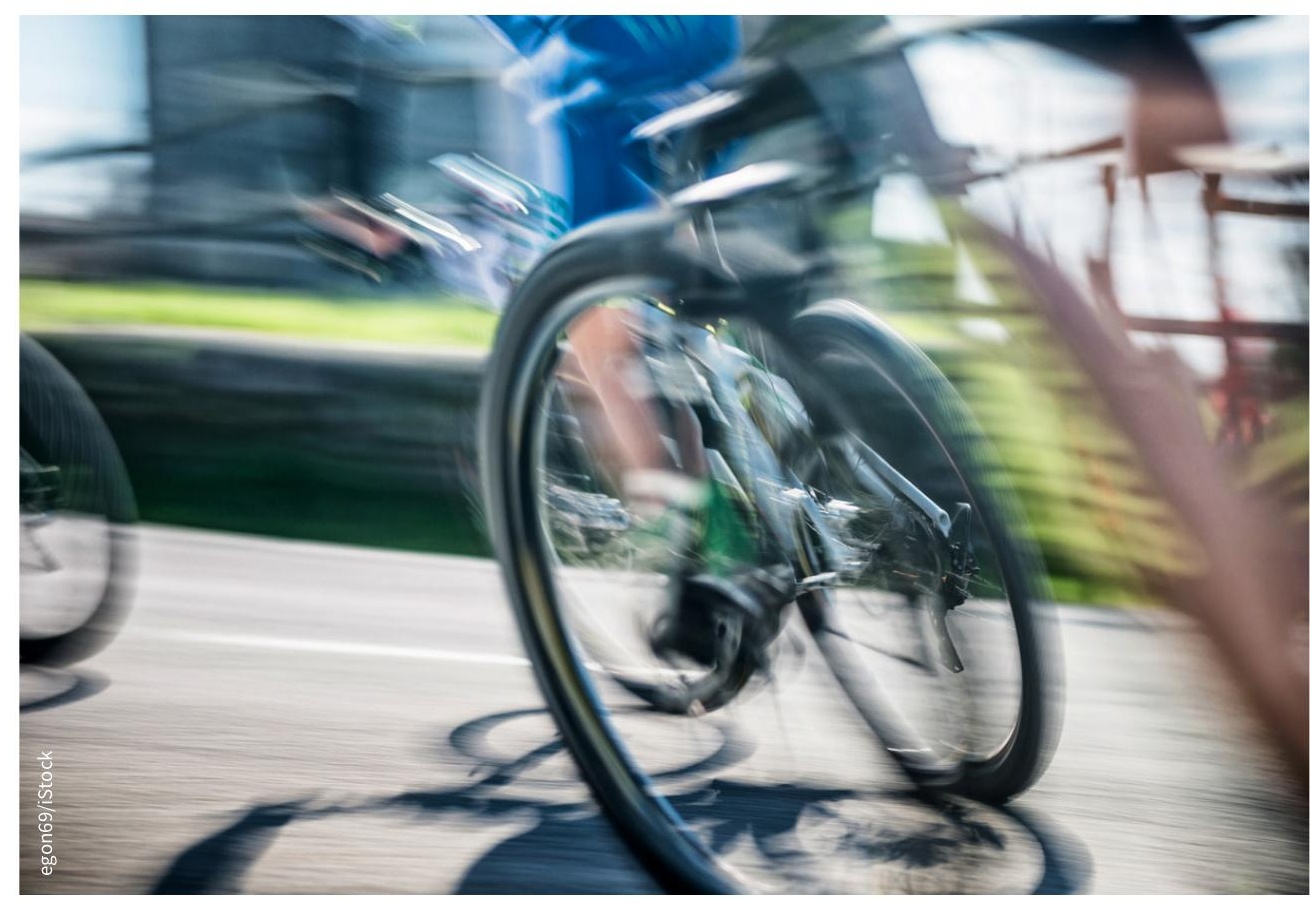

studying, which limits opportunities after graduation, and stopped taking care of my body. Oncology appointments were a hassle and distressing. Furthermore, knowing that I had tumours made it easy to start attributing symptoms to them: pain from a fractured pelvis, fatigue from too little sleep, feverish from a cold, dizzy from low blood pressure and so on. All of these symptoms reinforced my fears. was given. I gained a supreme appreciation for life and the little things in it. In some ways, this new attitude helped me through the very real, substantial injury I sustained a month after being cleared by my oncologist.

While out for a winter run, I slipped on some ice and fell, damaging ligaments and snapping all three bones in my ankle. After a very painful 12 hours in the emergency 
department, I had an x-ray and was told I needed an emergency operation. Five days later, I underwent surgery. It was five months before I was off crutches - the first time. After the second operation, I was discharged with chronic pain and severely restricted mobility that affected my daily life. No one knew why I wasn't getting better.

I sought out appointments with various specialists, but usually an x-ray, CT scan or MRI was required before consideration. Many times, I was told my situation was quite dismal; yet, wait times to get scans and then appointments with specialists who might have been able to help could take years. It felt like I was ferociously trying to fight my way back into the medical system that had once pulled me under its wing and zapped and examined me silly to confirm and reconfirm my health status. Why could't I find that kind of care now that I had a very real, actionable health problem?

On especially hard days - well, honestly, on most days - I wondered how many people ahead of me in the queue were being investigated knowingly or unknowingly for "phantom" problems as a result of incidentalomas and overdiagnosis. I am certainly not an expert on how the health care system works, but I do know that if I had broken my ankle on the oppo- site side of the city, I would have ended up back in the very same orthopedic ward where I used to see my oncologist.

Now that I am studying to become an epidemiologist, I often wonder what would happen if the resources, time and money spent on overdiagnosed health issues were reallocated toward identifying, diagnosing and treating people with health issues that can be helped. In my case, between ages 18 and 23, I had at least 11 bouts of $x$-rays, five CT scans, two MRIs, one bone scan, many blood tests, three orthopedic surgeries and countless appointments with various physicians. Only a portion of that was necessary. If my incidentalomas had not been investigated with such vigour, perhaps somebody with a dangerous yet treatable cancer could have gotten care a little bit sooner, or care that was a little bit better.

I could be telling a very different story. One where a bike crash led to the chance discovery of early-stage bone cancer and life-saving early interventions. Sure, that is possible and would be amazing. But that is not what happened.

My story is that one accident caused a fractured pelvis, which led to a lot of quick, yet unwanted and unneeded medical care, while a second accident caused the "worst possible" type of ankle fracture, which was followed by slow yet des- perately needed medical care. I continue to live with the consequences of incidentaloma investigation and thinking I had cancer. Upon discharge, I was told to come back if I experienced an increase in pain or other symptoms; even now, every illness or random hip pain makes me wonder if the tumours might be growing again. I would like to believe that my story is an oddity, but the more I learn, the more I realize it is not.

Fortunately, recent movements such as Choosing Wisely Canada, are encouraging doctors and patients to consider the potential harms around unnecessary medical tests. If a shared decision-making model had been used, or if someone had explained to me what incidentalomas are and the risks associated with investigating them, I might have understood my situation better and chosen not to have so many follow-up appointments unless "real" symptoms presented themselves. I might have been spared a lot of pain and emotional turmoil.

\section{Jacqueline Kathleen Kueper BSc}

Department of Epidemiology and

Biostatistics, Western University, London, Ont.

This article has been peer reviewed.

This is a true story. 